\title{
Status report of the upgrade of the CMS muon system with Triple-GEM detectors
}

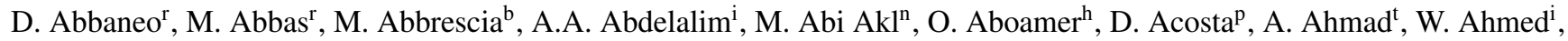

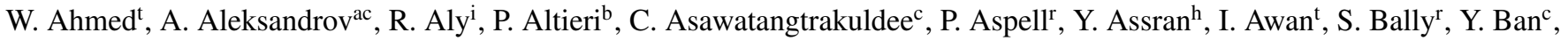

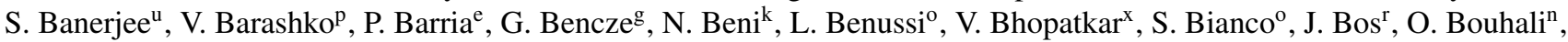
A. Braghieri ${ }^{\text {aa }}$, S. Braibant ${ }^{\mathrm{d}}$, S. Buontempo ${ }^{\mathrm{z}}$, C. Calabria ${ }^{\mathrm{b}}$, M. Caponero $^{\mathrm{o}}$, C. Caputo $^{\mathrm{b}}$, F. Cassese ${ }^{\mathrm{z}}$, A. Castaneda ${ }^{\mathrm{n}}$,

S. Cauwenbergh ${ }^{\mathrm{s}}$, F.R. Cavallo ${ }^{\mathrm{d}}$, A. Celik ${ }^{\mathrm{j}}$, M. Choi ${ }^{\mathrm{ag}}$, S. Choi ${ }^{\mathrm{ae}}$, J. Christiansen $^{\mathrm{r}}$, A. Cimmino ${ }^{\mathrm{s}}$, S. Colafranceschi $^{\mathrm{r}}$, A. Colaleo $^{\mathrm{b}}$, A. Conde Garcia ${ }^{r}$, S. Czellar ${ }^{\mathrm{k}}$, M.M. Dabrowskir ${ }^{\mathrm{r}}$, G. De Lentdecker ${ }^{\mathrm{e}, *}$, R. De Oliveirar ${ }^{\mathrm{r}}$, G. de Robertis ${ }^{\mathrm{b}}$, S. Dildick $^{\mathrm{j}, \mathrm{s}}$, B. Dorney ${ }^{\mathrm{r}}$,

W. Elmetenawee ${ }^{i}$, G. Endroczi ${ }^{\mathrm{g}}$, F. Errico ${ }^{\mathrm{b}}$, A. Fenyvesi ${ }^{\mathrm{k}}$, S. Ferry ${ }^{\mathrm{r}}$, I. Furic ${ }^{\mathrm{p}}$, P. Giacomelli ${ }^{\mathrm{d}}$, J. Gilmore , V. Golovtsov $^{\mathrm{q}}$,

L. Guiducci ${ }^{\mathrm{d}}$, F. Guilloux ${ }^{\mathrm{ab}}$, A. Gutierrez ${ }^{\mathrm{m}}$, R.M. Hadjiiskac ${ }^{\mathrm{ac}}$, A. Hassan ${ }^{\mathrm{i}}$, J. Hauser ${ }^{\mathrm{w}}$, K. Hoepfner ${ }^{\mathrm{a}}$, M. Hohlmann ${ }^{\mathrm{x}}$, H. Hoorani ${ }^{\mathrm{t}}$,

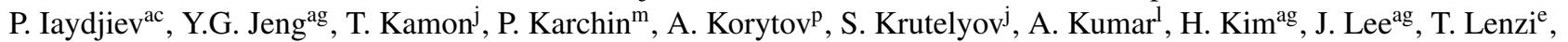

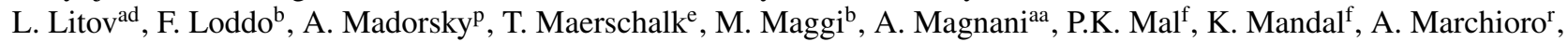
A. Marinov ${ }^{\mathrm{r}}$, R. Masod ${ }^{\mathrm{h}}$, N. Majumdar ${ }^{\mathrm{u}}$, J.A. Merlin ${ }^{\mathrm{r}, \mathrm{ah}}$, G. Mitselmakher ${ }^{\mathrm{p}}$, A.K. Mohanty ${ }^{\mathrm{y}}$, S. Mohamed $^{\mathrm{h}}$, A. Mohapatra $^{\mathrm{x}}$, J. Molnar ${ }^{\mathrm{k}}$, S. Muhammad ${ }^{\mathrm{t}}$, S. Mukhopadhyay ${ }^{\mathrm{u}}$, M. Naimuddin' ${ }^{1}$ S. Nuzzo ${ }^{\mathrm{b}}$, E. Oliveri ${ }^{\mathrm{r}}$, L.M. Pant ${ }^{\mathrm{y}}$, P. Paolucci ${ }^{\mathrm{z}}$, I. Park ${ }^{\mathrm{ag}}$,

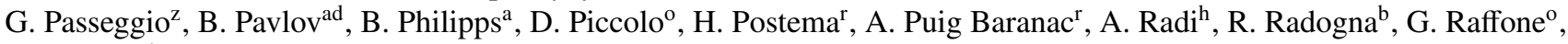

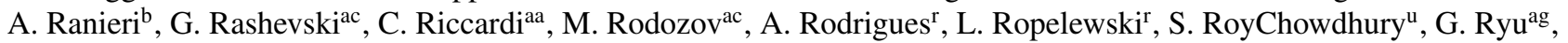
M.S. Ryu ${ }^{\mathrm{ag}}$, A. Safonov ${ }^{\mathrm{j}}$, S. Salva ${ }^{\mathrm{s}}$, G. Saviano ${ }^{\mathrm{o}}$, A. Sharma ${ }^{\mathrm{b}}$, A. Sharma ${ }^{\mathrm{r}}$, R. Sharma ${ }^{1}$, A.H. Shah ${ }^{1}$, M. Shopova ${ }^{\text {ac }}$, J. Sturdy ${ }^{\mathrm{m}}$, G. Sultanov ${ }^{\text {ac }}$, S.K. Swain ${ }^{\mathrm{f}}$, Z. Szillasi ${ }^{\mathrm{k}}$, J. Talvitie ${ }^{\mathrm{v}}$, A. Tatarinov ${ }^{\mathrm{j}}$, T. Tuuva ${ }^{\mathrm{v}}$, M. Tytgat ${ }^{\mathrm{s}}$, I. Vai ${ }^{\text {aa }}$, M. Van Stenis ${ }^{\mathrm{r}}$, R. Venditti ${ }^{\mathrm{b}}$,

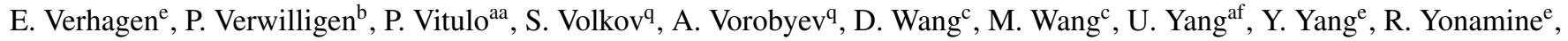
N. Zaganidiss, F. Zenoni ${ }^{\mathrm{e}}$, A. Zhang

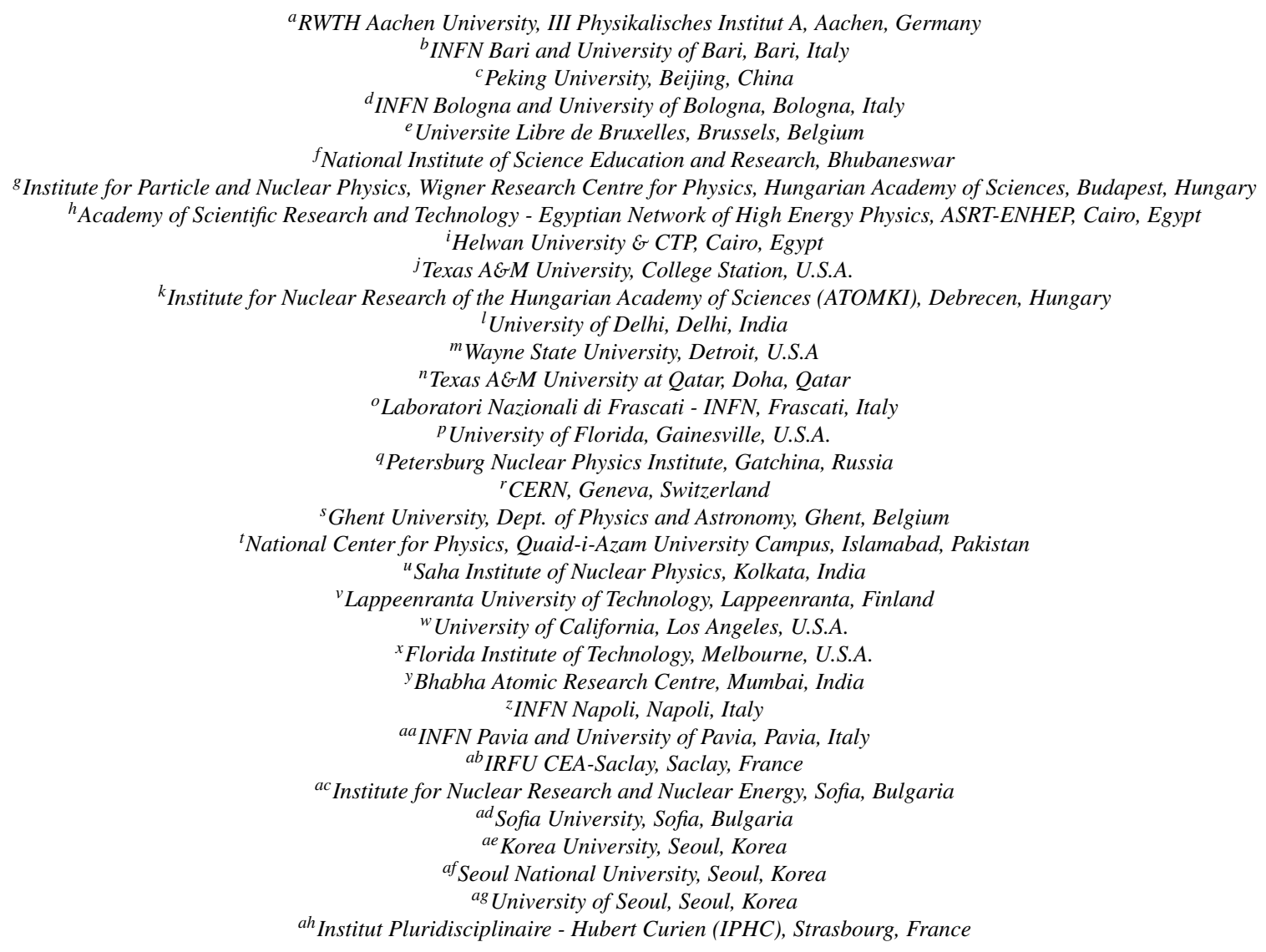

\begin{abstract}
For the High Luminosity LHC CMS is planning to install new large size Triple-GEM detectors, equipped with a new readout system, in the forward region of its muon system $(1.5<|\eta|<2.2)$. In this note we report on the status of the project, the main achievements regarding the detectors as well as the electronics and readout system.
\end{abstract}

Reprint sybmitted El Elsevier ${ }^{2}$ upgrades, GEM, Micro-Pattern Gas Detectors

November 19, 2015 PACS: 29.40.Cs, 29.40.Gx 


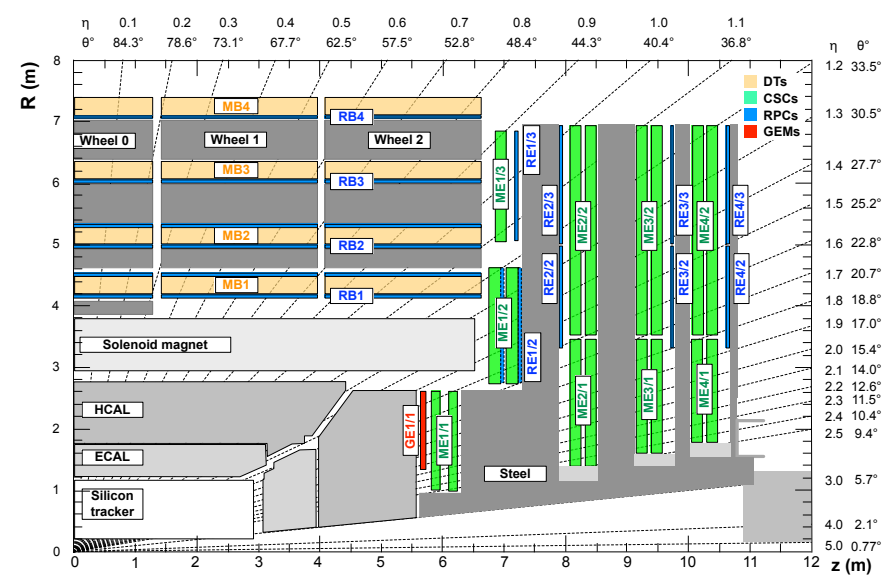

Figure 1: A quadrant of the $R-z$ cross-section of the CMS detector, highlighting in red the location of the proposed GE1/1 detector within the CMS muon system.

\section{Introduction}

For the High Luminosity LHC (HL-LHC), the CMS collab- 32 oration aims for the installation during the second LHC long ${ }^{33}$ shutdown (2018-2019) of an entire ring of Triple-GEMs [1], ${ }^{34}$ referred to as GE1/1, in the first endcap station of the CMS ${ }^{35}$ forward muon spectrometer, as shown in Figure 1. The TripleGEM system will re-establish the redundancy of the muon spec- ${ }^{36}$ trometer which, in the region $1.5<|\eta|<2.2$, is only equipped ${ }^{37}$ with Cathode Strip Chambers (CSC).

This notes summarizes the status of the GE1/1 project, un- ${ }^{39}$ derlining some of the main achievements regarding the detector as well as the electronics and the new readout system. The ${ }^{40}$ GE1/1 project schedule and the plans for additional CMS up- ${ }^{41}$ grades with Triple-GEMs are also briefly described.

\section{Requirements on GE1/1 detector performance and de- ${ }_{44}$} sign

To be installed inside the first station of the CMS forward ${ }^{46}$ muon spectrometer, the new detectors have to fulfil numerous requirements related to detection performance as well as mechanic and electronic constraints since these detectors have to be integrated inside an existing infrastructure.

- The new detectors should provide the largest geometric ac- ${ }^{49}$ ceptance within the given available envelope to improve ${ }^{50}$ as much as possible the physics yield. Figure 2 shows a ${ }^{51}$ picture of a $\sim 120 \mathrm{~cm}$ long GE1/1 Triple-GEM . The maxi- 52 mum thickness available in CMS is $9.9 \mathrm{~cm}$ which allows to 53 install back-to-back two Triple-GEMs, forming a so-called 54 superchamber. Each superchamber spans $10^{\circ}$ in $\phi$.

- With a $97.0 \%$ detection efficiency per Triple-GEM, a su- ${ }_{57}^{56}$ perchamber will have an efficiency above $99.9 \%$ when the ${ }_{58}$ signals from the two Triple-GEMs are 'ORed'.

${ }^{*}$ Corresponding author

Email address: gdelentd@ulb.ac.be (G. De Lentdecker)

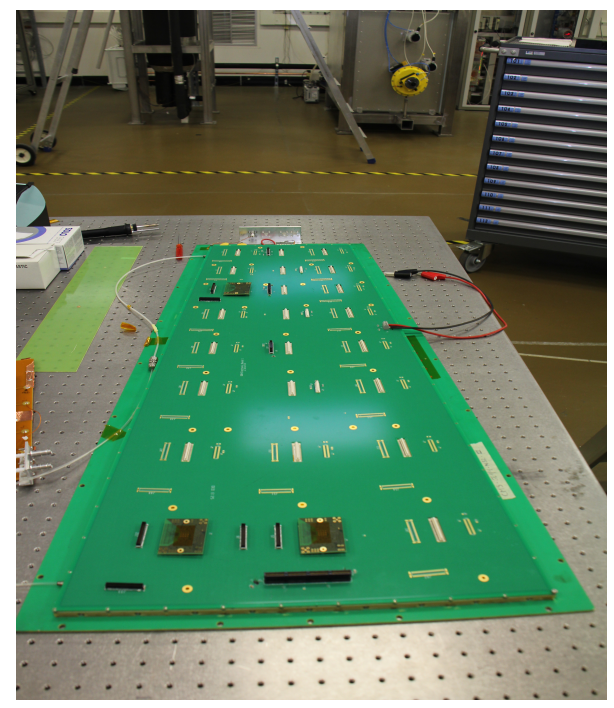

Figure 2: A CMS GE1/1 Triple-GEM detector equipped with the GEB PCB.

- Simulations have shown that for the CMS muon trigger an azimuthal resolution of $300 \mu \mathrm{rad}$ is sufficient. For a binary readout, this corresponds to a resolution of $0.8 \mathrm{~mm}$ at the outer radius $(2.6 \mathrm{~m})$ of the GE1/1 detectors.

- A time resolution of $10 \mathrm{~ns}$ or better per individual chamber, combined with the timing provided by the CSC located just behind, is sufficient to unambiguously match the muon to the correct LHC bunch crossing.

- The maximum expected hit rate within the GE1/1 acceptance is about a few $\mathrm{kHz} / \mathrm{cm}^{2}$ for HL-LHC running at 14 $\mathrm{TeV}$ and $5 \times 10^{34} \mathrm{~cm}^{-2} \mathrm{~s}^{-1}$.

- Finally the Triple-GEM detector response should be uniform within $10-15 \%$ and the detectors must be able to tolerate an accumulated ionization charge of $200 \mathrm{mC} / \mathrm{cm}^{2}$ to remain operational over 20 years of HL-LHC.

\section{Detector developments}

Over 5 years of intensive R\&D, the GE1/1 project introduced 6 generations of large-area Triple-GEM detectors; each generation built on the experience from the previous ones and bringing new improvements. In this note we will only review a couple of the main achievements.

In 2010 we introduced the first 1m-class Triple-GEM detector ever constructed and operated [2]. The various components were glued and spacers were used to keep the GEM foils apart. The third generation, which is shown in Figure 2 was the first detector in which the foils were stretched purely mechanically against the outer detector frame, but glue was still used to hold the different pieces of the frame and to glue it to the drift board [3]. In the fourth generation, both readout and drift boards were bolted to the outer frames and sealed with $\mathrm{O}$ rings making this generation, the first large-area GEM detector 


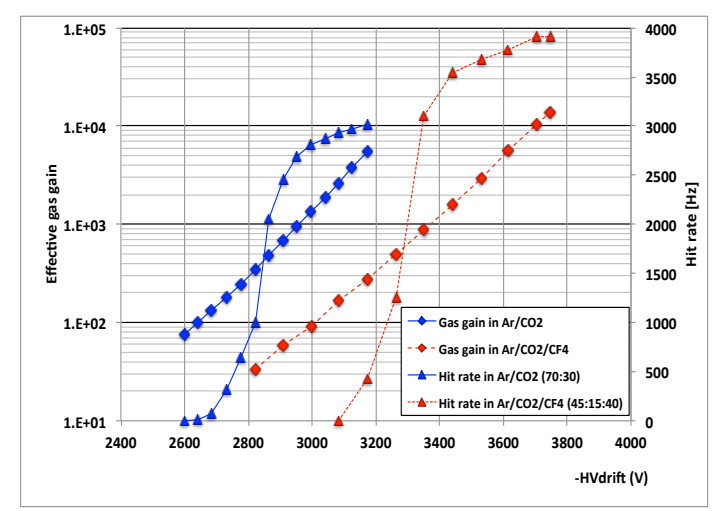

Figure 3: GE1/1 Triple-GEM gain as a function of the high voltage applied to the drift electrode for both $\mathrm{Ar} / \mathrm{CO}_{2} / \mathrm{CF}_{4}$ 45:15:40 and $\mathrm{Ar} / \mathrm{CO}_{2}$ 70:30 gas mixtures. The counting rate is also shown for a fixed rate of incident $\mathrm{X}$-rays.

produced without gluing any components. Consequently the as- ${ }^{99}$ sembly time could be reduced to a few hours. In the fifth gener- ${ }^{100}$ ation the assembly technique has been improved by tensioning the foils against independent "pull-out" pieces, onto which both readout and drift boards are bolted. The outer frame is made from a single piece and only serves to close the gas volume. It is sealed against readout and drift boards with O-rings. The ${ }^{104}$ last generation presents only minor modifications with respect ${ }^{105}$ to the fifth generation, mainly details to optimize the geometric ${ }^{106}$ acceptance within the CMS envelope. Note that although no ${ }^{107}$ glue is used in the assembly, every component in contact with ${ }^{108}$ the gas will be tested for ageing and/or outgassing.

\section{Performance}

The performance of the different GE1/1 prototypes have been ${ }^{113}$ studied thoroughly in laboratories as well as in a series of beam tests at CERN [2] - [4] and at Fermilab [5]. Only a few results are presented here. Most of the measurements are performed ${ }_{115}$ with the $\mathrm{Ar} / \mathrm{CO}_{2} / \mathrm{CF}_{4}$ 45:15:40 and $\mathrm{Ar} / \mathrm{CO}_{2}$ 70:30 gas mixtures. While for the GE1/1 project a new front-end chip, the VFAT3, ${ }^{116}$ is being developed (see section 5), the prototypes are most of ${ }^{117}$ the time read out with the binary VFAT2 [6] chips.

\subsection{Gas gain}

Figure 3 shows the gain of a 4 th generation GE1/1 Triple- ${ }^{120}$ GEM as a function of the high voltage applied to the drift elec-121 trode for both $\mathrm{Ar} / \mathrm{CO}_{2} / \mathrm{CF}_{4}$ 45:15:40 and $\mathrm{Ar} / \mathrm{CO}_{2}$ 70:30 gas $^{122}$ mixtures. The counting rate is also shown for a fixed rate of ${ }^{123}$ incident X-rays, featuring the beginning of rate plateaus which ${ }^{124}$ indicates that the chamber starts operating with full efficiency. The Triple-GEM starts to be efficient at a gas gain of several ${ }^{125}$ thousands. At this gain, the individual GEM foils are operated ${ }^{126}$ with a potential difference around $\sim 400 \mathrm{~V}$, providing a comfortable safety margin [7].

\subsection{Detection efficiency and spatial resolution}

Several detection efficiency measurements have also been ${ }^{129}$ performed with various prototypes showing that the GE1/1 $1_{130}$

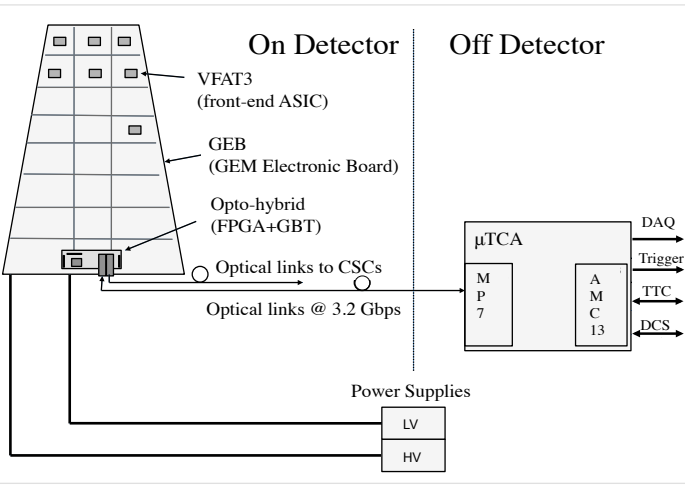

Figure 4: Readout architecture of the CMS GE1/1 system. both gas mixtures [5]. In Ref [5] a detailed study on the spatial resolution also shows that the GE1/1 Triple-GEMs have a spatial resolution of $\sim 137 \mu \mathrm{m}$ at a radius of $1.88 \mathrm{~m}$, which is consistent with the expected resolution for binary readout of $\sim 131 \mu \mathrm{m}$.

\subsection{Time resolution}

The timing performance first measured with $10 \times 10 \mathrm{~cm}^{2}$ prototypes has showed that using the $3 / 1 / 2 / 1 \mathrm{~mm}$ drift/transfer1/transfer-2/induction gap configuration and $\mathrm{Ar} / \mathrm{CO}_{2} / \mathrm{CF}_{4}$ 45:15:40 the Triple-GEM could reach time resolutions as good as $4 \mathrm{~ns}$. Since then the measurements have been confirmed with the full-size GE1/1 prototype [3]. It is important to note that even with the slower but environmentally-friendly $\mathrm{Ar} / \mathrm{CO}_{2}$ 70:30 gas mixture, an overal time resolution of $8 \mathrm{~ns}$ for a superchamber can be obtained, which is sufficient for the CMS trigger application.

\section{Readout system}

To control and read-out this new CMS sub-detector we propose the electronics system shown in Figure 4.

\subsection{The on-detector electronics}

The functional requirements on the readout system (and therefore on its front-end electronics) are to provide both triggering and tracking information. Similar functionalities are provided by the VFAT2. Therefore the VFAT2 architecture is the baseline for the new front-end ASIC, the VFAT3. The features of this new ASIC are listed below:

- Provide trigger data at $40 \mathrm{MHz}$ as well as precise tracking data upon Level-1 Accept (L1A) signal;

- Relatively long signal charge collection $\sim 80 \mathrm{~ns}$;

- Programmable shaping time: 25, 50, 100, 250, $500 \mathrm{ns;}$

- Interface required: slvds elinks to GBT [8] at $320 \mathrm{Mbps}$;

- Level-1 latency up to $20 \mu \mathrm{s}$; 
- Integrated calibration, bias and monitoring functions.

Similarly to VFAT2 it will have 128 channels of preampli- ${ }_{186}$ fier, shaper and comparator. Both trigger and tracking data are $_{187}$ sent to the off-detector electronics located in the CMS service ${ }_{188}$ cavern via the new Versatile Link [9]. The Versatile Link is bi- ${ }_{189}$ directional and operates at a rate of $4.8 \mathrm{Gbps}$. On-detector the ${ }_{190}$ GBT radiation hard chipset will transmit the data from the de- ${ }_{191}$ tector through the Versatile Link. The VFAT3 chip will embed ${ }_{192}$ an e-Port [10] to be connected directly to the GBT chipset. In $_{193}$ addition the trigger data will be sent to the CSC trigger electron- ${ }_{-194}$ ics to be combined with the CSC data to improve the Level- $1_{195}$ trigger efficiency of the CSC system.

The readout strips are oriented radially along the long side of ${ }_{197}$ the detector. The strip pattern is segmented in $8 \times 3$ partitions $_{198}$ in $\eta-\phi$. The strips are connected through metalized vias to the ${ }_{199}$ outer side of the readout board where 128-channel connectors ${ }_{200}$ are soldered for the signal transfer to the VFAT3. To avoid long $_{201}$ cables running along the detector the VFAT3 signals are trans- 202 mitted through the GEM Electronics Board (GEB). The $\mathrm{GEB}_{203}$ board is a $1 \mathrm{~mm}$ thick 6-layer PCB of the size of the detec-204 tor. Actually it is the green side of the detector seen in Fig- 205 ure 2. Electrical measurements have been done to characterize ${ }_{206}$ the signal integrity at $40 \mathrm{MHz}$ and the functionality of the GEB board with the VFAT2 chips has been tested successfully. Using pulse generators, the signal integrity has also been checked ${ }^{207}$ at $160 \mathrm{MHz}$ and work is now on-going to reach $320 \mathrm{MHz}$.

The last on-detector electronics component is the optohybrid which consists in a mezzanine board mounted along the large side of the GEB board, with typical dimensions of $10.0^{211}$ $\mathrm{cm} \times 20.0 \mathrm{~cm} \times 1.1 \mathrm{~cm}$. The tasks of the opto-hybrid board are to synchronize the data sent by the VFAT3 chips, zero-suppress the trigger data, encode the data and send them via optical links ${ }^{212}$ to the trigger electronics. The opto-hybrid is composed of a Virtex-6 FPGA, 3 GBT chipsets and 12 optical connectors of type SFP+ (small form factor pluggable).

\subsection{The off-detector electronics}

The off-detector electronics provides the interfaces from the ${ }^{219}$ detector (and front-end electronics) to the CMS data acquisi- ${ }_{221}$ tion (DAQ), the CMS Trigger Timing and Control (TTC) and 222 the Trigger systems. The design foreseen for the CMS GEM ${ }^{223}$ off-detector electronics is based on FPGAs boards with Multi- ${ }_{225}$ GBit/s links that adhere to the micro-TCA ( $\mu$ TCA) standard. ${ }_{226}^{225}$ In CMS $\mu$ TCA is now a common standard for all the CMS 227 upgrades and will replace the VME electronics. The GE1/1228 project benefits from $\mu \mathrm{TCA}$ boards developed by other $\mathrm{CMS}_{230}^{229}$ upgrades (trigger and HCAL).

\subsection{Schedule and Conclusions}

In December 2014, for the first time, a GE1/1 Triple-GEM ${ }^{235}$ detector equipped with the VFAT2 chips and with the new elec- ${ }_{237}^{236}$ tronics architecture, as described above, has been tested with ${ }_{238}$ particle beams at CERN. The aims of these tests were to val-239 idate the system architecture and to test as many electronics ${ }^{240}$ functionalities as possible. Performance measurements like noise measurements and cluster size have been performed and showed similar behaviour than with the former readout system.

In 2015, the developments are focusing on finalizing the various components for the readout of the VFAT2 chips, with the objectives to install in CMS four GE1/1 superchambers during the LHC extended yearly technical stop of 2016-17. The first two final GE1/1 detectors have been produced and the first GE1/1 superchamber is being assembled at the time of this writing.

Once the VFAT3 and the GBT chipsets will be available end of 2015, the developments will then focus on the final system: 72 GE1/1 superchambers will be installed in CMS during the second LHC long shutdown (2018-2019).

For the longer term, CMS plans to install in 2022-2023, a second station of Triple-GEM detectors, called GE2/1. The architecture of GE2/1 will be very similar than GE1/1 but with even larger detectors, spanning $20^{\circ}$ in $\phi$. R\&D is currently ongoing to design such large chambers. Finally, with the CMS plans to redesign the calorimeter endcap, there would be space to add a new muon tagging station, called ME0, in the region $2.0<|\eta|<3.0$ to enhance the far forward muon coverage. A possibility which is under study is to use stacks of six GE1/1like Triple-GEM detectors to reduce the neutron background.

\section{Acknowledgments}

We gratefully acknowledge support from FRS-FNRS (Belgium), FWO-Flanders (Belgium), BSF-MES (Bulgaria), BMBF (Germany), DAE (India), DST (India), INFN (Italy), NRF (Korea), QNRF (Qatar), and DOE (USA).

\section{References}

[1] F. Sauli, GEM: A new concept for electron amplification in gas detectors, Nucl. Instrum. Meth. A386 (1997) 531-534.

[2] D. Abbaneo, et al., Characterization of GEM Detectors for Application in the CMS Muon Detection System, IEEE Nucl. Sci. Symp. Med. Imag. Conf. Rec. (2010) 1416-1422.

[3] D. Abbaneo, et al., Beam Test Results for New Full-scale GEM Prototypes for a Future Upgrade of the CMS High-eta Muon System, IEEE Nucl. Sci. Symp. Med. Imag. Conf. Rec. (2012) 1172-1176.

[4] D. Abbaneo, et al., Test beam results of the GE1/1 prototype for a future upgrade of the CMS high- $\eta$ muon system, IEEE Nucl. Sci. Symp. Med. Imag. Conf. Rec. (2011) 1806-1810.

[5] D. Abbaneo, et al., Performance of a Large-Area GEM Detector Prototype for the Upgrade of the CMS Muon Endcap System, IEEE Nucl. Sci. Symp. Med. Imag. Conf. Rec. (2014).

[6] P. Aspell, et al., VFAT2: A front-end system on chip providing fast trigger information, digitized data storage and formatting for the charge sensitive readout of multi-channel silicon and gas particle detectors, Topical Workshop on Electronics for Particle Physics, Prague, Czech Republic, 03-07 Sept., pp. 292296. 2007

[7] F. Sauli, High rate behavior and discharge limits in micro-pattern detectors, Nucl. Instrum. Meth. A424 (1999) 321-342.

[8] P. Moreira, et al., The GBT SerDes ASIC prototype, 2010 JINST 5 C11016

[9] J. Troska al., Versatile Transceiver developments, 2011 JINST 6 C01089

[10] P. Aspell, et al., The VFAT3-Comm-Port: a complete communication port for front-end ASICs intended for use within the high luminosity radiation environments of the LHC, JINST 10 (2015) 03, C03019

[11] P. Aspell, et al., Development of a GEM Electronic Board (GEB) for triple-GEM detectors, JINST 9 (2014) 12, C12030 